
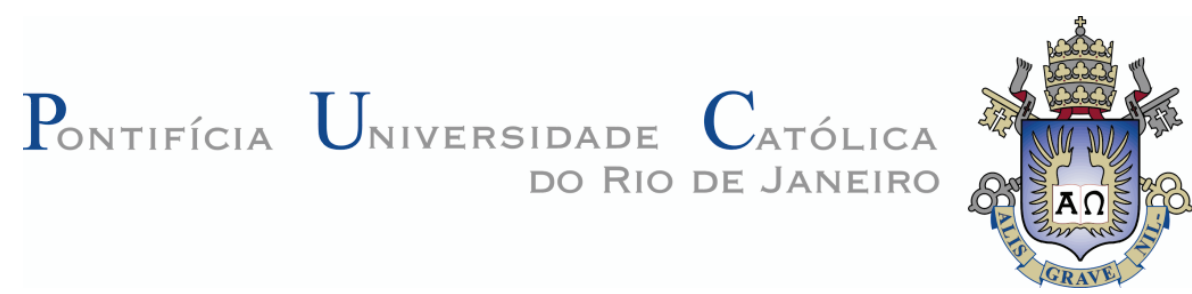

Andrés Benjamín Paladines Andrade

\title{
Modelo Inteligente de Avaliação da Qualidade de Água e da Qualidade Ambiental para um Reservatório Tropical Oligo-mesotrófico.
}

Dissertação de Mestrado

Dissertação apresentada como requisito parcial para obtenção do título de Mestre pelo Programa de PósGraduação em Engenharia Elétrica da PUC-Rio.

Orientador: Profa. Marley María Bernárdez Rebuzzi Vellasco Co-orientador: Profa. Christina Wyss Castelo Branco 


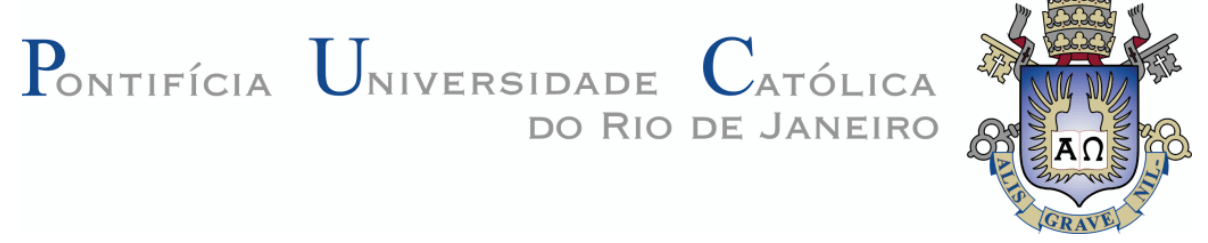

Andrés Benjamín Paladines Andrade

\section{Modelo Inteligente de Avaliação da Qualidade de Agua e da Qualidade Ambiental para um Reservatório Tropical Oligo-mesotrófico.}

Dissertação apresentada como requisito parcial para obtenção do título de Mestre pelo Programa de PósGraduação em Engenharia Elétrica da PUC-Rio. Aprovada pela Comissão Examinadora abaixo assinada.

Profa. Marley María Bernárdez Rebuzzi Vellasco

Orientador

Departamento de Engenharia Elétrica - PUC-Rio

Profa. Christina Wyss Castelo Branco

Co-Orientador

UNIRio

Profa. Karla Tereza Figueiredo Leite

UESO

Prof. Ricardo Tanscheit

Departamento de Engenharia Elétrica - PUC-Rio

Profa. Elisabete Fernandes Albuquerque Palermo

UNIRio

Prof. José Eugenio Leal Coordenador Setorial do Centro

Técnico Científico

Rio de Janeiro, 23 de agosto de 2013 
Todos os direitos reservados. É proibida a reprodução total ou parcial do trabalho sem autorização da universidade, do autor e do orientador.

\section{Andrés Benjamín Paladines Andrade}

Graduou-se em Engenharia Elétrica pela Universidade San Francisco de Quito - Equador 2009.

Ficha Catalográfica

Andrade, Andrés Benjamín Paladines

Modelo inteligente de avaliação da qualidade de água e da qualidade ambiental para um reservatório tropical oligo-mesotrófico / Andrés Benjamín Paladines Andrade ; orientador: Marley Bernárdez Rebuzzi Vellasco; co-orientador: Christina Wyss Castelo Branco. - 2013. 229 f. : il. (color.) ; $30 \mathrm{~cm}$

Dissertação (mestrado)-Pontifícia Universidade Católica do Rio de Janeiro, Departamento de Engenharia Elétrica, 2013.

Inclui bibliografia

1. Engenharia elétrica - Teses. 2. Índice de qualidade da água. 3. Índice ambiental. 4. Ferramenta para gestão de águas. 5. Ambiente lêntico. 6. Reservatório das lajes. 7. Sistema de inferência Fuzzy I. Vellasco, Marley Bernárdez Rebuzzi. II. Castelo Branco, Christina Wyss. III. Pontifícia Universidade Católica do Rio de Janeiro. Departamento de Engenharia Elétrica. IV. Título.

CDD: 621.3 
Para meus pais, Elsa e Iván. 


\section{Agradecimentos}

À Deus por ter me guiado nesta jornada.

À CAPES e á PUC-Rio pelos auxílios concedidos, sem os quais este trabalho não poderia ter sido realizado.

À Profa. Dra. Marley Maria B. R. Vellasco e à Profa. Dra. Christina Wyss Castelo Branco pelo apoio, incentivo e confiança.

Aos meus pais Elsa e Iván por estarem sempre presentes ao meu lado, me apoiando-me ao longo da minha vida.

À minha irmã Natalia pela ajuda e bons conselhos.

À minha Juliana pelo amor, carinho, apoio e, principalmente, pela paciência e compreensão ao longo da elaboração desta tese. 


\section{Resumo}

Andrade, Andrés Benjamín Paladines; Vellasco, Marley María Bernárdez Rebuzzi. Modelo Inteligente de Avaliação da Qualidade da Agua e da Qualidade Ambiental para um Reservatório Tropical Oligomesotrófico. Rio de Janeiro, 2013. 229 p. Dissertação de Mestrado Departamento de Engenharia Elétrica, Pontifícia Universidade Católica do Rio de Janeiro.

Uma forma de avaliar a qualidade da água e a qualidade ambiental de um reservatório para monitoramento futuro é listar e analisar as concentrações de tudo o que a mesma tem. Tal lista poderia ser tão longa quanto o número de elementos analisados, podendo ir de 20 e poucos componentes comuns a centenas. É assim que vários índices de qualidade têm sido propostos por serem capazes de sintetizar o maior número destes parâmetros de qualidade em um único valor de fácil interpretação. Não obstante, uma vez que a maior parte dos índices formulados serem para águas moventes, os mesmos têm pouca utilidade para lagos e reservatórios. Lagos e reservatórios são geralmente avaliados e classificados com base em índices de estado trófico e em análises de suas composições químicas. Porém, um índice de estado trófico não tem a mesma representatividade de um índice de qualidade, visto que o termo qualidade sugere uma avaliação subjetiva, importante ressaltar essa distinção de conceitos. Excelente ou pobre, a referência de qualidade da água depende do seu uso e das atitudes locais das pessoas. A definição de estado trófico e seu índice correspondente deveriam permanecer neutros a tais julgamentos subjetivos, mantendo-se numa estrutura dentro da qual podem ser feitas várias avaliações da qualidade da água. Dessa forma, no presente trabalho, criou-se um modelo de avaliação da qualidade da água e da qualidade ambiental para um reservatório tropical oligo-mesotrófico (reservatório das Lajes) capaz de representar em uma escala numérica as gradações nos níveis de qualidade, além de levar em consideração a subjetividade implícita no conceito de qualidade. A subjetividade da avaliação em discussão motivou o emprego da Lógica Fuzzy, metodologia capaz de representar, de forma mais eficiente e clara, os limites dos intervalos de variação dos parâmetros de qualidade para um conjunto de categorias subjetivas, quando esses limites não são bem definidos ou são imprecisos. Assim, foi desenvolvida uma ferramenta computacional baseada em Sistemas de Inferência 
Fuzzy que avalia automaticamente a qualidade em função de variáveis físicas, químicas e biológicas do reservatório. O referido modelo foi desenvolvido com base no conhecimento de especialistas em qualidade de água e qualidade ambiental do Centro de Ciências Biológicas e da Saúde da Universidade Federal do Estado do Rio de Janeiro (UNIRIO) e do Departamento de Biologia Animal da Universidade Federal Rural do Rio de Janeiro (UFRRJ). O modelo foi avaliado utilizando dados de coleta do reservatório das Lajes coletados no ano 2005, 2008 e 2009.

\section{Palavras-chave}

Índice de qualidade da água; Índice Ambiental; Ferramenta para Gestão de Águas; ambiente lêntico; Reservatório das Lajes; Sistema de Inferência Fuzzy. 


\section{Abstract}

Andrade, Andrés Benjamín Paladines; Vellasco, Marley María Bernárdez Rebuzzi (Advisor). Evaluation Intelligent Model of Water and Environmental Quality for a Tropical Oligo-mesotrophic Reservoir. Rio de Janeiro, 2013. 229 p. MSc. Dissertation - Departamento de Engenharia Elétrica, Pontifícia Universidade Católica do Rio de Janeiro.

There are many approaches to monitor the water and environmental qualities of a reservoir. One approach is to list and analyze the concentration of chemicals and physical characteristics that the amount of water it contains. Such a list could be as long as the number of elements analyzed, from a few common components to hundreds. Thus, many indices have been proposed since they are able to synthesize as many of these quality parameters into a single value for an easy interpretation. However, majority of the indices are formulated to evaluate lentic ecosystems, they have little use for lakes and reservoirs. Lakes and reservoirs are generally evaluated and classified based on trophic state indices and chemical composition analysis. Nevertheless, a trophic state index does not have the same representativeness of a quality index. The term quality implies a subjective judgment that is best kept separate from the concept of trophic state. Excellent or poor, water quality depends on the use of that water and the local attitudes of the people. The definition of trophic state and its corresponding index should remain neutral to these subjective judgments, remaining a framework within which various evaluations of water quality may be made. Accordingly, in today's world of technology and advancement there exists a unique model to evaluate water quality and environmental quality for a tropical oligo-mesotrophic reservoir which is located and known as the reservoir of Lajes in the State of Rio de Janeiro, Brazil. This model is capable of representing quality levels on a numerical scale gradation, and also takes into consideration the subjectivity implicit in the concept of quality. The subjectivity, implicit in the concept of quality, motivated the use of fuzzy logic. This is a methodology to represent more efficiently the limits of ranges of quality parameters for a set of subjective categories, when these limits are not well defined or are inaccurate. As a result, we developed a computational tool based on a Fuzzy Inference System that automatically assesses the quality in terms of the physical, chemical and 
biological characteristics of the reservoir. The model was developed based on the knowledge of experts on water quality and environmental quality from the Biological Sciences and Health Center of Universidade Federal do Estado do Rio de Janeiro (UNIRIO) and from the Department of Animal Biology of the Universidade Federal Rural do Rio de Janeiro (UFRRJ). The model was evaluated with data from the Lajes reservoir during the years 2005, 2008 and 2009.

\section{Keywords}

Water Quality Index; Environmental Index; Water Management; lentic ecosystem; Lajes Reservoir; Fuzzy Inference System. 


\section{Sumário}

1 Introdução $\quad 39$

1.1. Apresentação 39

1.2. Objetivo Geral 43

1.3. Objetivos Específicos 43

1.4. Descrição do trabalho 44

1.5. Contribuições 44

1.6. Organização do trabalho 45

2 Fundamentos $\quad 47$

2.1. Características limnológicas consideradas relevantes na elaboração do modelo $\quad 47$

2.1.1. Processos físicos em Lagos, Reservatórios e Rios 47

2.1.2. Represas Artificiais $\quad 57$

2.2. Índices de Qualidade e Índices de Estado Trófico 62

2.2.1. Indicadores de Qualidade 62

2.2.2. Índices de Qualidade de Água e Índices de Estado Trófico $\begin{array}{ll}\text { Tradicionais } & 63\end{array}$

2.2.3. Índices Fuzzy de Qualidade de Água e Índices Fuzzy de Estado Trófico.

2.3. Modelagem Fuzzy $\quad 80$

2.3.1. Conjuntos Fuzzy 82

2.3.2. Variáveis Linguísticas 86

2.3.3. Sistema de Inferência Fuzzy (SIF) 87

3 Formulação do Modelo Inteligente 93

3.1. Área de Estudo 93

3.1.1. Descrição da área de estudo - o Reservatório de Lajes 93

3.1.2. O trabalho de (SOARES et al., 2008) 95

3.1.3. Locais de amostragem $\quad 97$

3.2. Metodologias de Cálculo 100 
3.4. Indicadores Físicos e Químicos de Qualidade de Água usados na implementação do Índice

3.4.1. Temperatura 105

3.4.2. Oxigênio Dissolvido (OD) 105

3.4.3. Potencial Hidrogeniônico $(\mathrm{pH}) \quad 107$

3.4.4. Clorofila-a

3.4.5. Série de Nitrogênio (nitrogênio orgânico, amônio, nitrato e nitrito)

3.4.6. Fósforo Total 109

3.4.7. Condutividade 110

3.4.8. Turbidez 110

3.5. Índice de Assembleia de Peixes em Reservatórios (IAPR) 111

3.6. Métricas de Assembleia de Peixes em Reservatórios 113

3.6.1. Número de Espécies Nativas 113

3.6.2. Número de Siluriformes Nativos 113

3.6.3. Número de Characiformes Nativos 113

3.6.4. Porcentagem de Biomassa de Espécies não Nativas 113

3.6.5. Dominância 114

3.6.6. Índice de Shannon 114

3.6.7. Número de Lambaris N 114

3.6.8. Número de Hypostomus Affinis 114

3.7. Determinação dos Subíndices 115

3.8. Base de Regras, Conjuntos Fuzzy e Funções de Pertinência 116

3.8.1. Base de Regras 116

3.8.2. Conjuntos Fuzzy e Funções de Pertinência 126

4 A Ferramenta Computacional 139

4.1.1. Aplicativo 140

4.1.2. Arquivo Excel "Base" 144

5 Resultados $\quad 147$

5.1. Teste de confiabilidade da ferramenta com MATLAB 147

5.1.1. Índice de qualidade da água 149 
5.1.2. Índice Biótico

5.2. Avaliação do Índice MIAQR-PALADINES de Qualidade da Água 161

5.2.1. Análise de resposta do modelo para cada variável

5.2.2. Teste do Índice MIAQR-PALADINES de Qualidade da Água utilizando dados reais

5.3. Avaliação do índice MIAQR-PALADINES de Qualidade Biótica 206

5.3.1. Subíndice A 209

5.3.2. Subíndice B 210

5.3.3. Subíndice C 211

5.3.4. Subíndice D 212

5.3.5. Subíndice E 213

5.3.6. Subíndice $F \quad 214$

5.3.7. Índice MIAQR-PALADINES Biótico 215

5.3.8. Índice MIAQR-PALADINES Biótico vs. IAPR 216

5.4. Discussão dos resultados 217

5.4.1. Teste da eficácia do modelo com MATLAB. 217

5.4.2. Avaliação do Índice MIAQR-PALADINES de Qualidade da Água 217

5.4.3. Avaliação do índice MIAQR-PALADINES de Qualidade Biótica 219

6 Conclusões 221

7 Trabalhos futuros 223

Referências Bibliográficas $\quad 225$ 


\section{Lista de figuras}

Figura 1. Estratificação térmica em lagos. 51

Figura 2. Relação entre a densidade da água e a temperatura. 52

Figura 3. Padrão estacional de estratificação e circulação vertical do Lago D. Helvécio, leste do Brasil. 53

Figura 4. Intrusão de águas na superfície ou no fundo de lagos e represas.

Figura 5. Zonas horizontais de um reservatório (Kimmer e Groeger, 1984) e alterações na extensão das zonas, vazão e padrão de mistura para diferentes valores de $\mathrm{R}$ (tempo de retenção).
A) $10<R<100$ dias;
B) $R R>100$ dias;
C) $R, 10$ dias.
61

Figura 6. Curvas de variação do oxigênio dissolvido.

Figura 7. Curvas Médias de Variação de Qualidade das Águas. $\quad 67$

Figura 8. Fluxograma de processo do INQA

Figura 9. Conjuntos oligotrófico, mesotrófico eutrófico da função membro para fósforo total.

Figura 10. Tendência de eutroficação avaliada utilizando avaliação sintética fuzzy para o Reservatório Fei-Tsui, Taiwan.

Figura 11. Função de pertinência do conjunto "Pessoas Altas" na teoria clássica dos conjuntos.

Figura 12. Função de pertinência do conjunto fuzzy "Pessoas Altas" na teoria dos conjuntos fuzzy.

Figura 13. Conjuntos pessoas baixas e pessoas altas $\quad 84$

Figura 14. Funções de pertinência crescente e decrescente 84

Figura 15. Função de pertinência trapezoidal 85

Figura 16. Função de pertinência triangular 85

Figura 17. Variável linguística $\quad 86$

Figura 18. Sistema de Inferência Fuzzy.

Figura 19. Estrutura básica do processo de Inferência Fuzzy do problema de qualidade da água. $\quad 89$

Figura 20. Fuzzificação. $\quad 89$ 
Figura 21. Diagrama de um sistema de inferência fuzzy completo

Figura 22. Mapa do Reservatório de Lajes mostrando os locais de amostragem.

Figura 23. Amostras físicas e químicas e biológicas 98

Figura 24. Amostras físicas e químicas e biológicas - zona 1

Figura 25. Amostras físicas e químicas e biológicas - zona $2 \quad 99$

Figura 26. Amostras físicas e químicas e biológicas - zona 3

Figura 27. Amostras físicas e químicas e biológicas - zona $4 \quad 100$

Figura 28. Seleção da metodologia de cálculo para a avaliação de qualidade de água e qualidade biótica

Figura 29. Fluxograma dos Subíndices que compõem o Índice de Qualidade da Água

Figura 30. Fluxograma dos Subíndices que compõem o Índice de Qualidade Biótica

Figura 31. Conjuntos fuzzy e funções de pertinência das variáveis: Temperatura e Oxigênio Dissolvido

Figura 32. Conjuntos fuzzy e funções de pertinência das variáveis: pH e Clorofila-a

Figura 33. Conjuntos fuzzy e funções de pertinência das variáveis: Nitrato e Fósforo Total

Figura 34. Conjuntos fuzzy e funções de pertinência das variáveis: Nitrito e Amônio

Figura 35. Conjuntos fuzzy e funções de pertinência das variáveis: Condutividade e Turbidez

Figura 36. Conjuntos fuzzy e funções de pertinência das variáveis:

Número de Espécies Nativas e Número de Siluriformes Nativos

Figura 37. Conjuntos fuzzy e funções de pertinência das variáveis:

Número de Characiformes Nativos e \% de Biomassa de

Espécies Nativas

Figura 38. Conjuntos fuzzy e funções de pertinência das variáveis:

Dominância e Índice de Shannon

Figura 39. Conjuntos fuzzy e funções de pertinência das variáveis:

Número de Indivíduos da Espécie Lambaris e Número de Hypostomus 
Affinis

Figura 40. Primeira versão da ferramenta computacional

Figura 41. Interface gráfica do aplicativo

Figura 42. Controles do aplicativo

Figura 43. Configuração dos Sistemas de Inferência Fuzzy (SIF)

Figura 44. Características Espaciais: Curva temperatura vs.

profundidade, Camadas de Estratificação Térmica e Zona Horizontal da amostra.

Figura 45. Zona onde são apresentados os índices finais com seus respectivos subíndices.

Figura 46. Vista geral da parte inicial do arquivo Excel

Figura 47. Arquivo Excel - Métricas Físicas e Químicas

Figura 48. Arquivo Excel - Métricas Biológicas

Figura 49. Arquivo Excel - Profundidade vs. Temperatura

Figura 50. Arquivo Excel - Localização Geográfica

Figura 51. Arquivo Excel - Regras (Ex: Epilímnio-Temperatura)

Figura 52. Arquivo Excel - Funções de Pertinência (Ex: EpilímnioTemperatura)

Figura 53. Diagrama da implementação do Índice de Qualidade de Água realizada em Simulink-MATLAB.

Figura 54. Resultado dos Subíndices que conformam o Índice de Qualidade da Água (MIAQR-PALADINES e MATLAB Simulink)

Figura 55. Resultado dos Subíndices que conformam o Índice de Qualidade da Água (MIAQR e MATLAB Simulink) (continuação)

Figura 56. Resultados do Índice de Qualidade da Água (MIAQR-

PALADINES e MATLAB Simulink)

Figura 57. Diagrama da implementação do Índice de Qualidade biótico realizada em Simulink-MATLAB

Figura 58. Resultado dos Subíndices que conformam o Índice Biótico (MIAQR-PALADINES e MATLAB Simulink)

Figura 59. Resultado dos Subíndices que conformam o Índice Biótico (MIAQR-PALADINES e MATLAB Simulink) (continuação) 
Figura 60. Resultados do Índice Biótico (MIAQR-PALADINES e

MATLAB Simulink)

Figura 61. Análise de comportamento do modelo - Variável

Temperatura

Figura 62. Análise de comportamento do modelo - Variável

Temperatura (continuação)

Figura 63. Análise de comportamento - Variável Oxigênio Dissolvido 166

Figura 64. Análise de comportamento - Variável Oxigênio Dissolvido (continuação)

Figura 65. Análise de comportamento - Variável pH 169

Figura 66. Análise de comportamento - Variável pH (continuação) 170

Figura 67. Análise de comportamento - Variável Clorofila-a

172

Figura 68. Análise de comportamento - Variável Clorofila-a (continuação)

Figura 69. Análise de comportamento - Variável Nitrato

Figura 70. Análise de comportamento - Variável Nitrato (continuação)

176

Figura 71. Análise de comportamento - Variável Fósforo Total

Figura 72. Análise de comportamento - Variável Fósforo Total (continuação)

Figura 73. Análise de comportamento - Variável Nitrito

Figura 74. Análise de comportamento - Variável Nitrito (continuação)

Figura 75. Análise de comportamento - Variável Amônio

Figura 76. Análise de comportamento - Variável Amônio (continuação)

Figura 77. Análise de comportamento - Variável Condutividade 187

Figura 78. Análise de comportamento - Variável Turbidez 189

Figura 79. Resultados do subíndice 1, ponto de coleta L1 ano 2009192

Figura 80. Resultados do subíndice 2, ponto de coleta L1, ano 2009192

Figura 81. Resultados do subíndice 3, ponto de coleta L1 ano 2009193

Figura 82. Resultados do subíndice 4, ponto de coleta L1, ano 2009193

Figura 83. Resultados do subíndice 5, ponto de coleta L1 ano 2009194 
Figura 84. Resultados do subíndice 6, ponto de coleta L1, ano 2009

Figura 85. Resultados do subíndice 7, ponto de coleta L1 ano 2009195

Figura 86. Resultados do subíndice 8, ponto de coleta L1, ano 2009195

Figura 87. Resultados do Índice MIAQR-PALADINES de Qualidade da Água, ponto de coleta L1, ano 2009

Figura 88. Resultados do subíndice 1, ponto de coleta L5, ano 2009198

Figura 89. Resultados do subíndice 2, ponto de coleta L2, ano 2009198

Figura 90. Resultados do subíndice 3, ponto de coleta L5, ano 2009199

Figura 91. Resultados do subíndice 4, ponto de coleta L5, ano 2009199

Figura 92. Resultados do subíndice 5, ponto de coleta L5, ano 2009200

Figura 93. Resultados do subíndice 6, ponto de coleta L5, ano 2009200

Figura 94. Resultados do subíndice 7, ponto de coleta L5, ano 2009201

Figura 95. Resultados do subíndice 8, ponto de coleta L5, ano 2009201

Figura 96. Resultados do índice MIAQR-PALADINES de Qualidade da Água, ponto de coleta L5, ano 2009

Figura 97. Resultado do índice final para os locais amostrais L2,

L3 e L4 , ano 2009

205

Figura 98. Avaliação do subíndice A 209

Figura 99. Avaliação do subíndice B 210

Figura 100. Avaliação do subíndice $C$

Figura 101. Avaliação do subíndice D 212

Figura 102. Avaliação do subíndice E 213

Figura 103. Avaliação do subíndice F 214

Figura 104. Avaliação do índice final 215

Figura 105. Resultados do Índice MIAQR-PALADINES Biótico vs. O IAPR 


\section{Lista de tabelas}

Tabela 1. Parâmetros que compõem o NSF-IQA 64

Tabela 2. Estado trófico como função do nível de nutrientes (OECD) 75

Tabela 3. Coordenadas dos locais de amostragem - variáveis físicas e químicas

Tabela 4. Coordenadas dos locais de amostragem - métricas de assembleia de peixes

Tabela 5. Critério de atribuição de notas - IAPR

Tabela 6. Avaliação da qualidade biótica conforme o valor do IAPR

Tabela 7. Variáveis Físicas e Químicas - dados de teste

Tabela 8. Resultado dos Subíndices que conformam o Índice de Qualidade da Água (MIAQR-PALADINES e MATLAB Simulink)

Tabela 9. Resultados do Índice de Qualidade da Água (MIAQR-

PALADINES e MATLAB Simulink)

Tabela 10. Variáveis Biológicas - dados de teste

Tabela 11. Resultado dos Subíndices que conformam o Índice Biótico (MIAQR-PALADINES e MATLAB Simulink)

Tabela 12. Resultados do Índice Biótico (MIAQR-PALADINES e MATLAB Simulink)

Tabela 13. Valores constantes dos indicadores segundo o teste

Tabela 14. Resultado dos subíndices e índice final caso os indicadores sejam constantes

Tabela 15. Influência da variável Temperatura nos subíndices 1,6 e

8, e no índice MIAQR-PALADINES de Qualidade da Água

Tabela 16. Influência da variável Oxigênio Dissolvido nos subíndices

1, 6 e 8, e no índice MIAQR-PALADINES de Qualidade da Água

Tabela 17. Influência da variável pH nos subíndices 2, 6 e 8, e no índice MIAQR-PALADINES de Qualidade da Água

Tabela 18. Influência da variável Clorofila-a nos subíndices 2, 6 e 8 , e no índice MIAQR-PALADINES de Qualidade da Água

Tabela 19. Influência da variável Nitrato nos subíndices 3,7 e 8 , 
e no índice MIAQR-PALADINES de Qualidade da Água

Tabela 20. Influência da variável Fósforo Total nos subíndices 3, 7

e 8, e no índice MIAQR-PALADINES de Qualidade da Água

Tabela 21. Influência da variável Nitrito nos subíndices 4,7 e 8, e no índice MIAQR-PALADINES de Qualidade da Água

Tabela 22. Influência da variável Amônio nos subíndices 4, 7 e 8, e no índice MIAQR-PALADINES de Qualidade da Água

Tabela 23. Influência da variável Condutividade nos subíndice 5 e no índice MIAQR-PALADINES de Qualidade da Água

Tabela 24. Influência da variável Turbidez nos subíndice 5 e no índice MIAQR-PALADINES de Qualidade da Água

Tabela 25. Indicadores Físicos e Químicos, ponto de coleta L1, ano 2009

Tabela 26. Resultados dos subíndices e índice final, ponto de coleta L1, ano 2009

Tabela 27. Indicadores Físicos e Químicos, ponto de coleta L5, ano 2009

Tabela 28. Resultados dos subíndices e índice final, ponto de coleta L5, ano 2009

Tabela 29. Resultado do índice final, ponto de coleta L2, ano 2009203

Tabela 30. Resultado do índice final, ponto de coleta L3, ano 2009203

Tabela 31. Resultado do índice final, ponto de coleta L4, ano 2009204

Tabela 32. Base de dados utilizada na avaliação do Índice MIAQR-

PALADINES Biótico

Tabela 33. IAPR, subíndices e índice MIAQR-PALADINES Biótico resultantes da avaliação

Tabela 34. Resultados do Índice MIAQR-PALADINES Biótico

vs. o IAPR 